\section{RMD Open}

Rheumatic \&

Musculoskeletal Diseases

\title{
Arthropathy of Down syndrome: an under-diagnosed inflammatory joint disease that warrants a name change
}

\author{
Charlene M Foley, ${ }^{\oplus, 2}$ Derek A Deely, ${ }^{1}$ Emma Jane MacDermott, ${ }^{1}$ Orla G Killeen ${ }^{1,2}$
}

To cite: Foley CM, Deely DA, MacDermott EJ, et al. Arthropathy of Down syndrome: an underdiagnosed inflammatory joint disease that warrants a name change. RMD Open 2019;5:e000890. doi:10.1136/ rmdopen-2018-000890

Received 26 December 2018 Revised 21 April 2019 Accepted 23 April 2019
Check for updates

(c) Author(s) (or their employer(s)) 2019. Re-use permitted under CC BY-NC. No commercial re-use. See rights and permissions. Published by BMJ.

${ }^{1}$ National Centre for Paediatric Rheumatology, Our Lady's Children's Hospital, Crumlin, Ireland

${ }^{2}$ National Children's Research Centre, Dublin, Ireland

Correspondence to Dr Charlene M Foley; charlenefoley@live.co.uk

\section{ABSTRACT}

There is an increased incidence and prevalence of arthropathy in children with Down syndrome. However, it is rarely reported or recognised at onset, and remains underdiagnosed. Children with arthropathy of Down syndrome (A-DS) are presenting with significant joint damage and disability at diagnosis.

Objective To identify undiagnosed cases of A-DS and document time to diagnosis. Also to describe clinical, laboratory and radiological features of A-DS at diagnosis. Methodology Children with Down syndrome (DS) (0-21 years) were invited to attend a musculoskeletal screening clinic. A second physician at a further clinic confirmed suspected cases of A-DS. Investigations and treatment were instigated as per normal clinical practice for Juvenile idiopathic arthritis (JIA). Data on a convenience sample of 21 newly diagnosed children with JIA was collected to create a comparison group.

Results Over an 18-month period, 503 children with DS were screened for arthritis and 18 new cases diagnosed. In total, 33 children were identified with A-DS (combining cases attending pre-dating commencement of the study and those referred to our centre during the study period). This suggests prevalence of A-DS is 20/1000. A significant delay in diagnosis of A-DS was observed. The majority of children presented with polyarticular-rheumatoid factornegative arthritis, with predominance in the small joints of the hands and wrists. Erosive changes were reported on $X$-ray in a significantly greater proportion (42\%) of children with A-DS than JIA (14\%). MRI was used to confirm diagnosis in four cases.

Conclusion Children with DS are at increased risk of arthritis. Future research to accurately define disease pathogenesis and identify a biomarker of disease would be of benefit.

\section{INTRODUCTION}

Juvenile idiopathic arthritis (JIA), an autoimmune disease, is an umbrella term used to describe seven different subtypes of childhood arthritis using the International League of Associations for Rheumatology (ILAR) classification criteria. ${ }^{1}$ It has an estimated European prevalence of 1 in $1000,{ }^{2}$ and an incidence of about $0.2-2.3$ per $10000 .^{3}$ Early diagnosis and aggressive treatment of JIA are

\section{Key messages}

What is already known about this subject?

- Children with Down syndrome (DS) are at increased risk of arthritis.

- Inflammatory arthritis in children with DS is most often polyarticular rheumatoid factor negative, with predominance in the small joints of the hands and wrists.

What does this study add?

- There is a general lack of awareness about the increased risk of arthritis in children with DS among the general public and healthcare professionals. This almost certainly contributes to the delayed diagnosis and irreversible joint damage observed in this cohort of children.

How might this impact on clinical practice?

- Inflammatory arthritis can be asymptomatic, and potentially erosive and debilitating if left undetected and untreated. An annual musculoskeletal assessment as part of physician guidelines for health surveillance in children with DS would help identify affected children.

- MRI with gadolinium contrast is a useful diagnostic tool for definitive diagnosis of arthritis in children with DS. Consider if any concerns, as clinically and laboratory-wise there can be little to aid diagnosis.

essential to maximise an optimal outcome. ${ }^{4-8}$ Delay or absence of appropriate treatment can result in potentially devastating consequences, including permanent disability from joint destruction, growth deformities and even blindness (from chronic uveitis associated with JIA). ${ }^{9}$

Down syndrome (DS) is a chromosomal disorder caused by full trisomy 21 (94\%$97 \%)$, translocations $(2 \%-4 \%)$ or mosaicism $(1 \%-2 \%) \cdot{ }^{10-15}$ It is a phenotypically heterogeneous condition with an estimated worldwide incidence of $1 / 1000$. Irish incidence is $1 / 547$, the highest in Europe. ${ }^{1617}$ DS is characterised by distinctive facial features, learning difficulties and a variety of medical conditions 


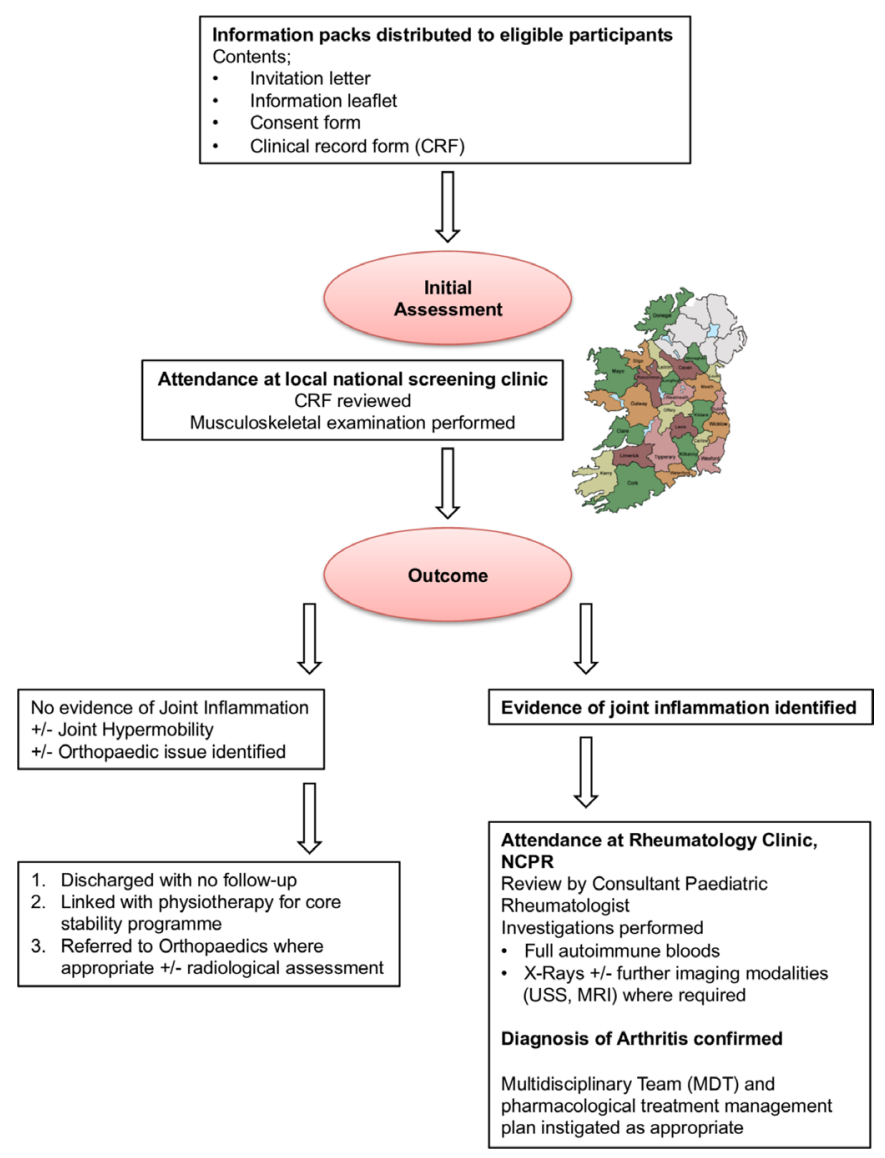

Figure 1 Flow diagram summarising the musculoskeletal screening process and follow-up provided to all children with Down syndrome that participated in the study.

including autoimmune disorders such as diabetes mellitus, coeliac disease and thyroid dysfunction. ${ }^{18-25}$ Arthritis is reported to occur, but is rarely recognised at onset, and remains under-diagnosed. ${ }^{26}$

Studies looking specifically at arthritis in DS are limited. All involve retrospective chart reviews. ${ }^{26-32}$ Other than two separate case reports by Sherk $e t a l^{27}$ and Herring $e t a l^{28}$ in 1982, Yancey et al. $(1984)^{30}$ were the first to describe in detail a JIA-like arthropathy in seven children with DS, and coined the phrase 'arthropathy of DS' (A-DS).

There are no published population surveys but crude estimates suggest more than a random association between DS and arthritis, with higher incidence and prevalence rates than JIA. A-DS incidence is reported to be threefold greater than JIA, and prevalence 8.7-10.2/1000. ${ }^{26} 2931$ Despite these suspected higher incidence and prevalence rates, a delay in diagnosis is a feature consistently reported in the few published reports to date. ${ }^{2630} 31$ Olson et al $(1990)^{31}$ report the mean time to diagnosis of A-DS was 3.3 years (range $0.25-9$ years). A recent study reports time to diagnosis of JIA from symptom onset was 0.25 years (0.02-6.8 years), significantly less than reported for children with A-DS. ${ }^{33}$ Delayed diagnosis of arthritis leads to both articular and extra-articular complications. ${ }^{34}$ In A-DS, the delay in diagnosis is potentially detrimental and could lead to permanent joint damage and disability in a population already at risk of gross motor limitations. ${ }^{35-37}$

The aim of this study was to identify cases of undiagnosed arthritis and delineate time to diagnosis of arthritis in children with DS. In addition, we describe clinical, laboratory and radiological features of A-DS at diagnosis.

\section{METHODS}

This was an observational study carried out over an 18-month period that involved the development of national musculoskeletal screening clinics for children with DS. The target population was children aged 0-21 years with DS living in the Republic of Ireland. Using EUROCAT (European surveillance of congenital anomalies) data, this was estimated to be 1652 individuals ( www.eurocat-network.eu). The target population was sourced using convenience sampling with the help of a national charity organisation, Down Syndrome Ireland (DSI); 1300 children registered with their service met the target population criteria. Other avenues used to source participants were general paediatric, specialist and developmental clinics attended by children with DS in our tertiary hospital (Our Lady's Children's Hospital, Crumlin (OLCHC) ); through liaison with the Chartered Physiotherapists in Intellectual Disability of Ireland; and by enrolment of the study in the Irish Paediatric Surveillance Unit (IPSU).

Awareness and information about the study were made available to as many as possible of the target population through the sources outlined above. Eligible participants were invited to attend local or regional musculoskeletal screening clinics where a full medical history was ascertained and a musculoskeletal examination performed by a paediatric rheumatology clinical fellow. If concerns were raised with regards to joint inflammation and/or new musculoskeletal findings, children were invited to attend the National Centre for Paediatric Rheumatology (NCPR) at OLCHC for assessment by a Consultant Paediatric Rheumatologist. At this appointment, diagnosis of arthritis was clinically confirmed, or appropriate referrals for radiological confirmation made if required. The active joint count (AJC), universally defined as the number of joints with clinical evidence of pain and/or swelling, or evidence of synovial enhancement on MRI with gadolinium contrast of the affected joint; and restricted joint count (RJC), universally defined as the number of joints with limitation of the normal range of movement expected were documented. Investigation and management were as per normal clinical practice for all newly diagnosed cases of arthritis. Bloods including C-reactive protein (CRP), erythrocyte sedimentation rate (ESR), anti-nuclear antibody (ANA) and rheumatoid factor $(\mathrm{RF})$, and baseline X-rays of affected joints were undertaken in cases identified. Figure 1 summarises the musculoskeletal screening process and follow-up provided to all children with DS that participated in the study. 
Table 1 Demographic, clinical and laboratory features at diagnosis of A-DS $(n=33)$ and JIA $(n=21)$. Where data are presented as median and range, statistical significance was determined using the Mann-Whitney $U$ test. For data presented as a proportion of the total population, statistical significance was calculated using the $\chi^{2}$ test or Fisher exact test

\begin{tabular}{llll}
\hline Characteristic & $\begin{array}{l}\text { A-DS } \\
\mathbf{n}=\mathbf{3 3}\end{array}$ & $\begin{array}{l}\text { JIA } \\
\mathbf{n}=\mathbf{2 1}\end{array}$ & $\mathbf{p}$ value \\
\hline Age, median in years (range) & 11.4 & 6.3 & $\mathrm{p}<0.001$ \\
& $(0.3-19.2)$ & $(1.1-16)$ & $\mathrm{ns}$ \\
Gender, $\mathrm{n}$ male (\% male) & $18(55)$ & $11(52)$ & $\mathrm{ns}$ \\
\hline AJC, $\mathrm{n}$ (range) & 3 & 4 & \\
& $(0-18)$ & $(1-13)$ & $\mathrm{p}<0.05$ \\
RJC, $\mathrm{n}$ (range) & 4 & 1 & \\
& $(0-12)$ & $(0-10)$ & $\mathrm{p}<0.01$ \\
*Small joint involvement, $\mathrm{n}(\%)$ & $28(85)$ & $9(43)$ & $\mathrm{p}<0.01$ \\
Raised ESR at diagnosis, $\mathrm{n}(\%)$ & $8 \dagger(32)$ & $15(71)$ & $\mathrm{ns}$ \\
\hline Raised CRP at diagnosis, $\mathrm{n}(\%)$ & $3 \neq(10)$ & $6(29)$ & $\mathrm{p}<0.01$ \\
\hline ANA positive, $\mathrm{n}(\%)$ & $0(0)$ & $5(24)$ & $\mathrm{ns}$ \\
\hline RF positive, $\mathrm{n}(\%)$ & $0(0)$ & $1(5)$ & \\
\hline
\end{tabular}

*Small joint involvement is defined as evidence of active inflammation in the metacarpophalangeal, proximal and/or distal interphalangeal joints of the hands.

†A-DS cohort of $n=25$.

$\ddagger A$-DS cohort of $n=29$ ( $n$ numbers less than total cohort as results missing).

A-DS, Arthropathy of Down syndrome; AJC, Active joint count;ANA, anti-nuclear antibody; CRP, C-reactive protein; ESR, erythrocyte sedimentation rate; JIA, Juvenile Idiopathic Arthritis ; RF, rheumatoid factor; RJC, Restricted joint count; $n$, number; ns, not significant.

A comparison group was created using a convenience sampling method of all children newly diagnosed with JIA at the NCPR over the same 18-month period. The OLCHC Ethics Committee granted approval for this study.

\section{RESULTS}

Over the 18-month period of the study, 503 children with DS underwent a detailed musculoskeletal examination. This equates to about $30 \%$ of the target population $(503 / 1652)$. Of the 503 children screened, $56 \%$ were male and the median age was 8.1 years $(0.6-19.2$ years). Combining cases detected through screening $(n=18)$, new cases directly referred to our centre during this time period $(n=4)$, and those diagnosed and attending our centre pre-dating the national screening initiative $(n=11)$, a total of 33 A-DS cases were recruited to the study. Data was also collected on 21 children newly diagnosed with JIA; $52 \%$ male, median age 6.3 years (1.1-16 years).

Demographic, clinical and laboratory features at diagnosis of the A-DS and JIA cohorts are presented in table 1. Time to diagnosis of A-DS was 1.2 years (0.1-4.5 years), significantly delayed $(\mathrm{p}<0.05)$ compared with JIA, 0.4 years $(0.1-3$ years) (figure $2 \mathrm{~A})$.

In the JIA cohort (figure $2 \mathrm{~B} / \mathrm{C}$ ), two peaks are seen with regards to age at diagnosis. One occurs in early infancy (48\%) and the other in the pre-teen years (48\%). Only $4 \%$ of children with JIA were diagnosed at 13 years or older. In contrast, the greatest percentage of children diagnosed with A-DS were between 13 and 20 years.

Of the 18 children detected through screening, $89 \%$ $(n=16)$ had poor language skills or were non-verbal, and
$28 \%(\mathrm{n}=5)$ were on the autistic spectrum. Only three $(17 \%)$ of the parents suspected arthritis, and confirmed that this suspicion arose only on reading the study information leaflet. Figure 3A presents the route taken to reach rheumatology for children with A-DS and JIA. The majority of children with JIA were referred to rheumatology by their paediatrician $(38 \%)$ or general practitioner (GP; 33\%). In contrast, over half of children diagnosed with A-DS (55\%) were detected through the national musculoskeletal screening initiative.

A conservative estimate of A-DS prevalence calculated using 33 cases known in an estimated target population of 1652 , is $20 / 1000$. This suggests that A-DS is at least 20 times more common than JIA. ${ }^{2}$

Applying the ILAR classification criteria to the A-DS cohort, the majority of children would be classified as the subtype, polyarticular-RF-negative arthritis (82\%) (figure 3B). This proportion is significantly higher than observed in the JIA cohort where $19 \%$ presented with this subtype $(\mathrm{p}<0.00001)$.

\section{Clinical and laboratory features of A-DS}

Figure 3C illustrates the joints affected by arthritis in A-DS. The proximal interphalangeal (PIP) joints of the hands were most frequently involved (79\%). Small joint involvement of the hands, which includes the metacarpophalangeal (MCP), PIP and distal interphalangeal (DIP) joints, was significantly more prevalent $(\mathrm{p}<0.01)$ in A-DS (85\%) than JIA (43\%). Furthermore, $30 \%$ of the cohort of children with A-DS presented with involvement of the small joints of the hands and/or wrist(s) only. 
A

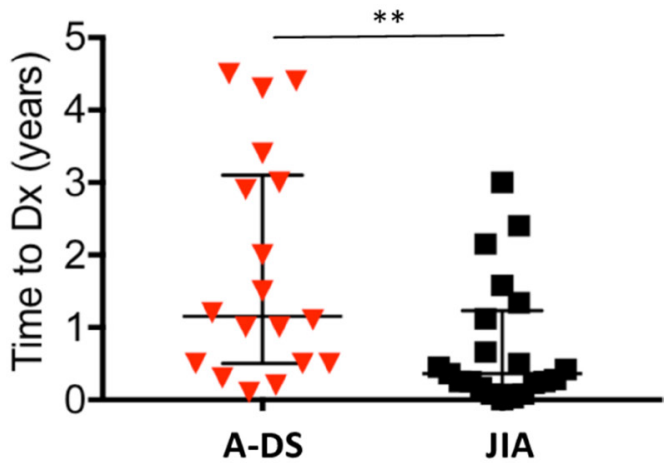

B

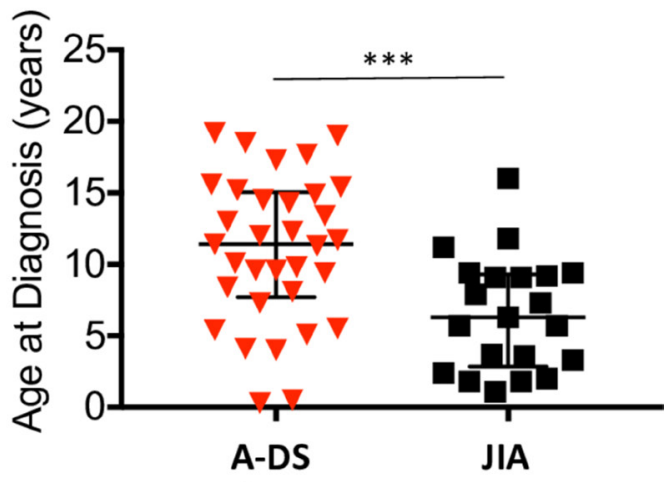

C

- A-DS Cohort $\square$ JIA Cohort

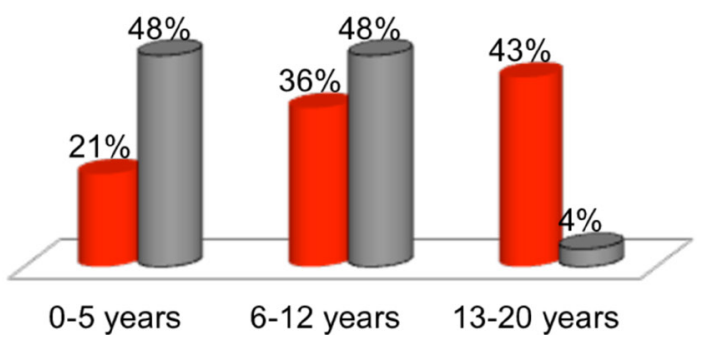

Figure 2 Time to diagnosis from symptom onset and age at diagnosis of A-DS and JIA. (A) The dot plot represents time to diagnosis (Dx) from symptom onset of A-DS $(n=18)$ and JIA $(n=21)$. Data are presented as median \pm interquartile range (IQR). Average time to diagnosis for children with A-DS was significantly delayed compared to children with JIA, determined using a Mann-Whitney $U$ test, ${ }^{*} p<0.05$, ${ }^{* *} p<0.01$, ${ }^{* * *} p<0.001$. (B) The dot plot represents age at diagnosis of A-DS $(n=33)$ and JIA $(n=21)$. Data are presented as median \pm IQR. Average age at diagnosis for children with A-DS was significantly older compared to children with JIA, determined using a Mann-Whitney U test, ${ }^{*} p<0.05,{ }^{* *} p<0.01$, ${ }^{* \star *} p<0.001$. (C) The bar chart represents the age distribution at diagnosis in the A-DS and JIA cohorts. Results are presented as percentage of total cohort (A-DS, $n=33$ and JIA, $n=21$ ). Two main peaks are observed in JIA. One in early infancy (48\%) and the second in the pre-teen years (48\%). In contrast, the greatest proportion of children with A-DS (43\%) were diagnosed at age 13 years or above. A-DS, arthropathy of DS; JIA, juvenile idiopathic arthritis.

A similar AJC was observed at diagnosis between the A-DS and JIA cohorts. This pattern was not reflected in the ESR and CRP measurements (figure 4A/B). Both acute phase reactants (APRs) were raised less frequently
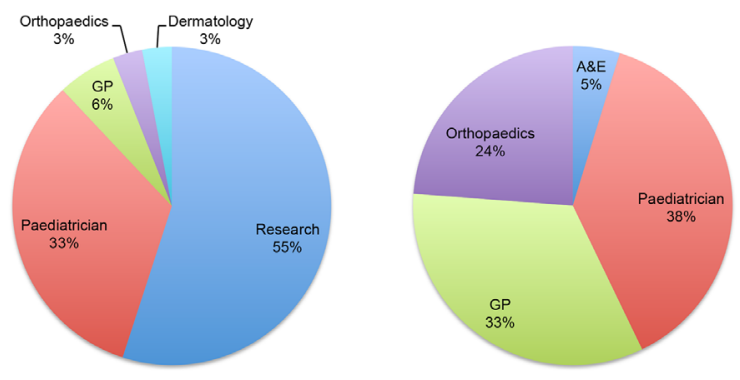

B
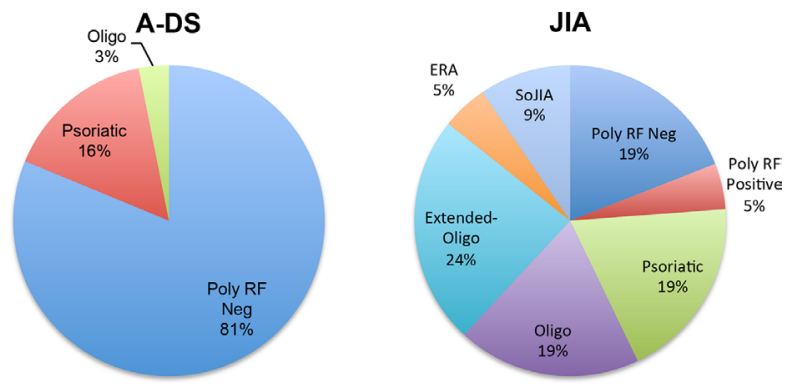

c

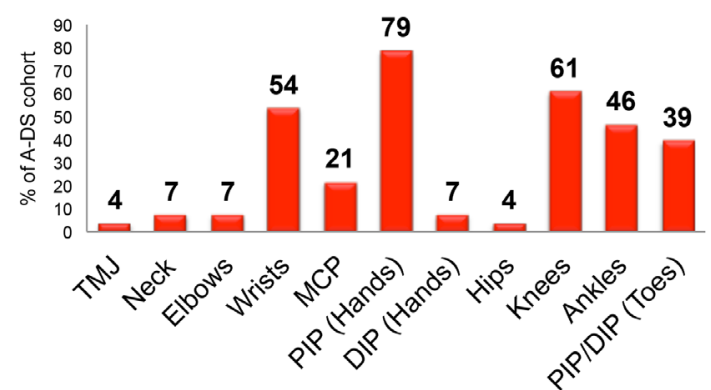

Figure 3 Route to rheumatology and ILAR classification of A-DS and JIA, plus presenting joints involved in A-DS. (A) The pie charts represent the route to rheumatology care for children diagnosed with A-DS $(n=33)$ and JIA $(n=21)$. Children with JIA are most commonly referred by their Paediatrician, GP or Orthopaedic team. In contrast, the majority of children diagnosed with A-DS were detected through the national musculoskeletal screening initiative (ie, research). (B) The pie charts represent the ILAR subtypes of arthritis observed in children diagnosed with A-DS $(n=33)$ and JIA $(n=21)$. (C) The bar chart represents the joints affected by arthritis at diagnosis in children diagnosed with A-DS. The most commonly affected joints in this cohort of children were the PIP joints of the hands. A-DS, arthropathy of DS; A\&E, accident and emergency department; ERA, enthesitis-related arthritis; GP, general practitioner; ILAR, International League of Associations for Rheumatology; JIA, juvenile idiopathic arthritis; MCP, metacarpophalangeal joint; Oligo, oligoarticular arthritis; PIP/DIP, proximal/ distal interphalangeal joint; Poly, polyarticular arthritis; RF, rhematoid factor; SoJIA, systemic-onset JIA; TMJ, temporomandibular joint.

in A-DS. A significantly greater RJC was observed in children with A-DS at diagnosis $(\mathrm{p}<0.05)$ (figure 4C).

ANA positivity (titres $>1 / 160$ ) occurred in $24 \%$ of the JIA cohort $(n=5)$. All children with A-DS had a negative ANA measurement. Over the time period of this study, no cases of uveitis have been identified in the A-DS cohort, with one case occurring in the JIA cohort (5\%). 
A

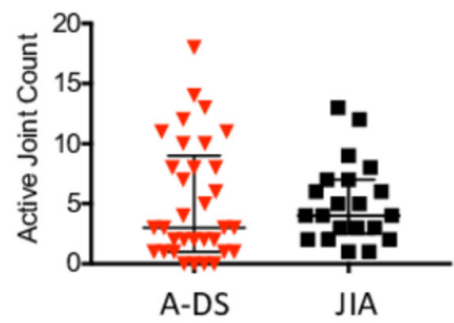

A-DS

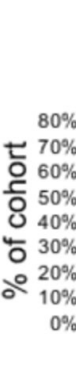

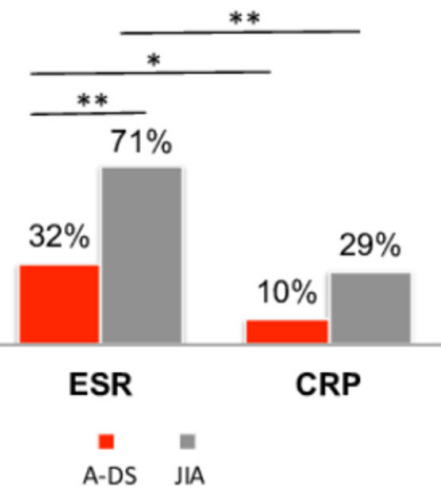
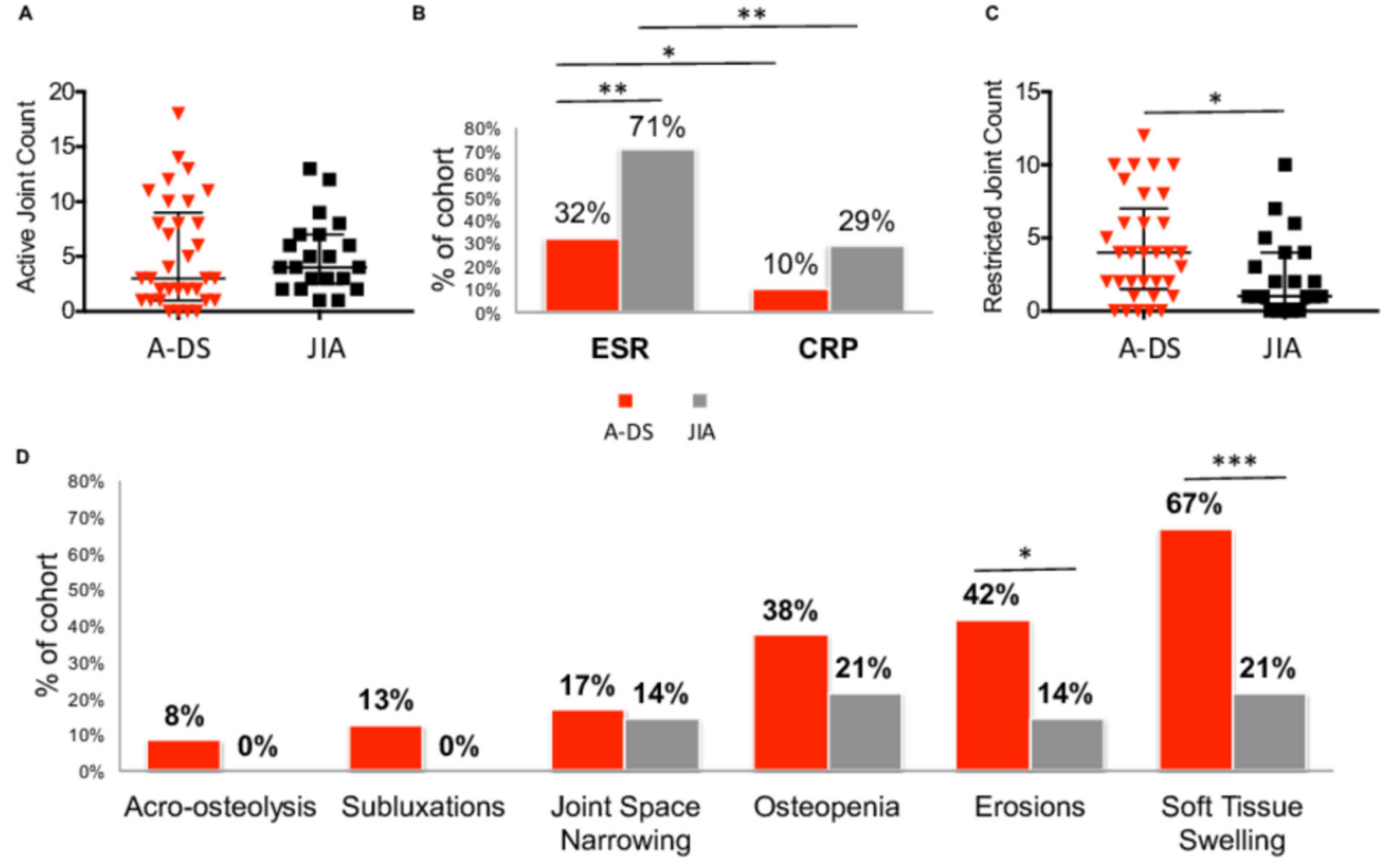

- A-DS = JA
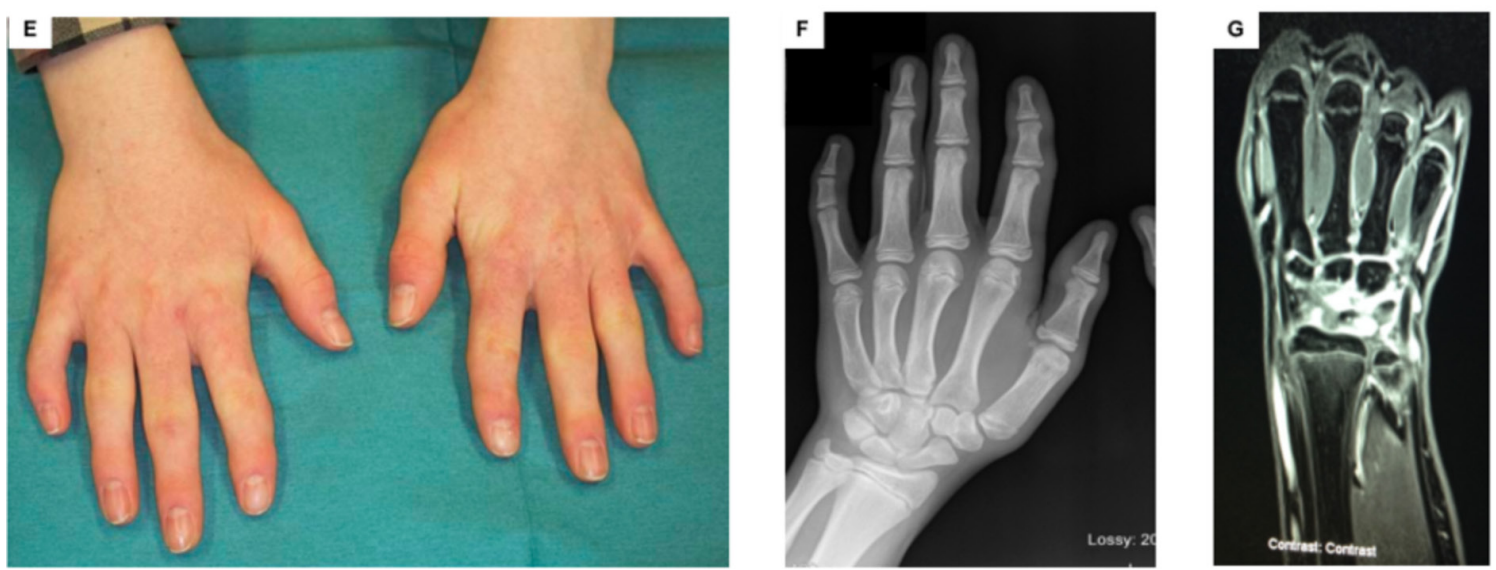

Figure 4 Clinical, laboratory and radiological features of arthritis at diagnosis in children with A-DS and JIA; and the importance of MRI with gadolinium contrast in aiding diagnosis of active synovitis in children with A-DS. (A) Dot plot represents AJC at diagnosis in the cohort of children with A-DS $(n=33)$ and JIA $(n=21)$. (B) The bar chart represents the proportion of children with A-DS and JIA that had raised APRs at diagnosis (ESR and CRP). (C) Dot plot represents RJC at diagnosis in the cohort of children with A-DS $(n=33)$ and JIA $(n=21)$. (D) Bar chart demonstrating features identified by a paediatric musculoskeletal radiologist on joint X-rays taken at diagnosis in children with A-DS and JIA. In (A) and (C), data are presented as median +/- IQR and statistical significance determined using a Mann-Whitney $U$ test, ${ }^{*} p<0.05,{ }^{* *} p<0.01$, ${ }^{* \star} p<0.001$. In (B) and (D), data are presented as a percentage of the total cohort and statistical significance calculated using the $\chi^{2}$ test, ${ }^{*} p<0.05,{ }^{* *} p<0.01,{ }^{* * \star} p<0.001$. (E) Photograph of the hands of a 13-year- old boy with DS diagnosed with arthritis. Clinodactyly of the fifth digits noted bilaterally. Thickening of the PIP joints of the index, middle and to a lesser degree ring fingers is noted bilaterally. The thumb IP joints are also thickened bilaterally, as is the left thumb MCP joint. This young boy was asymptomatic on examination, although there was slight loss of end range of movement on flexion of the left wrist. Features were consistent with a history of previous arthritis with no concurrent active disease. (F) AP radiograph of the left hand of the boy described in figure 4E demonstrates some soft tissue swelling overlying the PIP and DIP joints of the index finger and thumb IP joint, with less prominent changes overlying the distal phalanx of the other fingers. Irregularity to the tip of the distal phalanx of the little finger represents a small erosion. (G) MRI with gadolinium contrast of the left wrist described in figure 4E and $F$ shows enhancement of the synovium overlying the proximal carpal row and MCP joints suggestive of active synovitis. A-DS, arthropathy of DS; AJC, active joint count; AP, anteroposterior; APRs, acute phase reactants; CRP, C-reactive protein; DIP, distal interphalangeal; DS, Down syndrome; ESR; erythrocyte sedimentation rate; IP, interphalangeal; JIA, juvenile idiopathic arthritis; MCP, metacarpophalangeal joint; PIP, proximal interphalangeal; RJC, restricted joint count. 


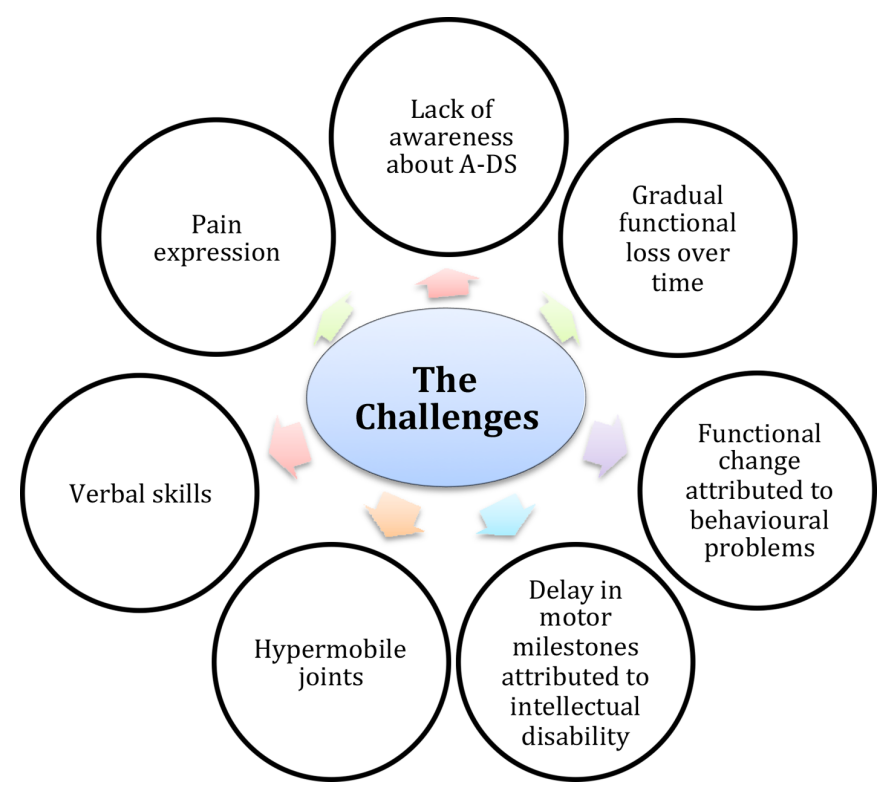

Figure 5 Schematic highlighting challenges which may impede correct and timely diagnosis of A-DS (from top, clockwise). The first is lack of awareness about the increased risk of arthritis in children with DS among both the general public and healthcare professionals; Gradual functional loss over time rather than an acute presentation may go undetected by the child's carer. Frequently, delay in motor development is falsely attributed to intellectual disability, and changes in activities of daily living to behavioural problems associated with DS, rather than a possible diagnosis of arthritis. Hypermobility, a feature of DS may make musculoskeletal examination more challenging, as it may be difficult to appreciate loss of range of movement secondary to an inflammatory arthritis. Many children may be uncooperative when it comes to examination. This combined with poor verbal skills can make eliciting a clear history and thorough musculoskeletal examination challenging. Apparent differences in pain expression have been reported in children with DS. Children with DS often adapt to pain with reported observations such as slowing mobility, reluctance to hold a parental hand or behavioural change. Therefore, these are key features to try and tease out when taking a history from a child with a suspected diagnosis of A-DS.

\section{Radiological features of A-DS}

A range of radiological features were observed in A-DS and JIA at diagnosis (figure 4D). Plain radiographs, reported by a paediatric musculoskeletal radiologist were performed on joints with clinical evidence of active arthritis, or joints that were deemed clinically inactive but had signs to suggest previous disease in the joint, such as bony articular thickening.

Soft tissue swelling was the most common feature reported, noted in $67 \%$ of children with A-DS, significantly more frequently $(\mathrm{p}<0.001)$ than in children with JIA (21\%). Erosions, suggesting irreversible joint damage were reported in $42 \%$ of children with A-DS imaged. This was significantly greater $(p<0.05)$ than the frequency of erosions identified in children with JIA (14\%).

MRI with gadolinium contrast was performed in 4/33 (12\%) children with suspected A-DS. All four patients had active synovitis confirmed with significant enhancement of the ulnocarpal, radiocarpal, intercarpal, carpometacarpal, MCP and PIP joints. No MRI scans were required to confirm diagnosis in the JIA cohort as clinical signs were sufficient.

\section{DISCUSSION}

Prior to commencement of this research, studies of arthritis associated with DS have been limited. ${ }^{26} 29-32$ In this report, we describe a cohort of 33 children with A-DS. Comparable to previous reports, our results confirm an increased risk of arthritis in children with DS. However, we suggest that prevalence is at least 2-3 times greater than previously reported. We also observed a significant delay in diagnosis of A-DS, the reasons for this being multifactorial.

Figure 5 highlights the number of challenges that may be encountered when caring for children with DS that may impede correct and timely diagnosis of A-DS. The first is the lack of awareness about the increased risk of arthritis in children with DS among both the general public and healthcare professionals. This is illustrated by the fact that the majority of parents whose child received a diagnosis of A-DS following attendance at a screening clinic were unaware that their child was displaying signs of arthritis. In fact, the delay in time to diagnosis we report for our cohort of A-DS may be an underestimation as $45 \%$ $(n=15)$ were unable to estimate time of symptom onset. These 15 cases were detected through the screening clinics. Lack of awareness can also contribute to misdiagnoses, a finding observed and reported by Cruikshank et al. (2008). ${ }^{38}$ In this study, the authors found that despite presenting on numerous occasions with clinical findings consistent with a diagnosis of arthritis, misdiagnoses such as soft tissue injury, multiple epiphysial dysplasia and development delay were made. Frequently delay in motor development is falsely attributed to intellectual disability, and changes in activities of daily living attributed to behavioural problems associated with DS, rather than a possible diagnosis of arthritis. ${ }^{31}$ Hypermobility, a feature of DS may make musculoskeletal examination more challenging, as it may be difficult to appreciate loss of range of movement secondary to an inflammatory arthritis. If there is gradual functional loss over time rather than an acute presentation, this may go undetected by the child's parent/guardian thus contributing to a delay in diagnosis. Many children may be uncooperative when it comes to examination. This combined with poor verbal skills can make eliciting a clear history and thorough musculoskeletal examination challenging. Interestingly, the majority of children with A-DS detected through the screening clinics $(89 \%)$ had poor language skills or were non-verbal, and $28 \%$ were on the autistic spectrum. This may explain why these children had not previously presented to their GP or paediatrician with joint concerns, as they may have been unable to articulate symptoms to their caregivers. 
Apparent differences in pain expression have also been reported in children with DS. Studies have shown that fewer responses to painful stimuli have been observed in laboratory mice with DS, individuals with DS express pain or discomfort more slowly and less precisely than the general population, and parents of children with DS report more difficulty identifying pain, or the source of their child's pain. ${ }^{39-41}$ Defrin et al (2004) ${ }^{42}$ suggest that individuals with DS are in fact more sensitive to pain than normal, but that slower reaction time gives the impression that pain threshold is higher than it actually is. With this understanding in mind, it is easy to see how differences in pain expression between children with and without DS may contribute to failure to report arthritis symptoms by children with A-DS. We identified that children with DS often adapt to pain with observations such as slowing mobility, reluctance to hold a parental hand or behavioural change reported by care givers. Therefore, a detailed history is required to identify potential clues that may aide diagnosis in suspected cases of A-DS.

The majority of children with A-DS presented with polyarticular-RF-negative arthritis, predominantly affecting the small joints of the hands and wrists. A high proportion had erosive changes at diagnosis, greater than observed in the JIA comparison group. This highlights that children with A-DS present with a greater degree of irreversible joint damage and disability at diagnosis. This may be as a consequence of the delay in diagnosis observed; however, our clinical impression is that A-DS appears to be a more aggressive, erosive disease than JIA such that children with A-DS are developing erosions earlier in their disease course than would typically be expected in a child with JIA.

Although JIA is defined as onset of arthritis before 16 years, the highest frequency occurs in children aged 1-3 years. ${ }^{2}$ Disease onset before 1 year is distinctly unusual in any category of JIA, and no cases less than 1 year were identified in our JIA cohort. Age at diagnosis in our JIA cohort was biphasic, occurring in the $0-5$ and 6-12 year age groups (figure 2C). The ILAR subtype, polyarticular-RF-negative JIA has two peaks in age of onset; the first in infancy (1-4 years) and the second at $6-12$ years. ${ }^{43}$ Applying the ILAR criteria to our cohort of children with A-DS, most children would be classified as having polyarticular-RF-negative arthritis. However, in children with A-DS, the age at diagnosis was significantly older compared with our JIA cohort, and did not demonstrate a biphasic peak in age of onset as would be expected for a typical cohort of children with a polyarticular-RF-negative pattern of arthritis. Furthermore, in contrast to the apparent older age of onset of arthritis in children with A-DS, two children with DS developed arthritis before age 1 , in fact both less than 6 months of age. It is likely that the older peak observed for age of diagnosis in the cohort of children with A-DS reflects delay to diagnosis. In fact, children with A-DS may develop arthritis at a younger age than would be expected for a child with JIA.
Clinical and laboratory features observed in A-DS appear to have some distinct differences to JIA. Eighty-one per cent of children with A-DS presented with polyarticular-RF-negative arthritis; this proportion being significantly greater than observed in the JIA cohort (figure 3B). Similar to our JIA cohort, in a global population of children with JIA, the ILAR subtype polyarticular JIA accounts for approximately $20 \%$, about $15 \%$ of whom would be RF positive. ${ }^{24}$ No children with A-DS were RF positive. Oligoarticular JIA $(50 \%-80 \%)$ is the most common form of childhood arthritis. ${ }^{2}$ This subtype was observed in only $3 \%$ of the A-DS cohort. The most commonly affected joint in JIA is a knee, whereas in A-DS the PIP joints of the hands $(79 \%)$ predominate (figure 3C). In some instances (30\%), children with A-DS presented with small joint and wrist involvement only. No children with A-DS were ANA positive or had developed uveitis at the time of publication. In a cohort of children with polyarticular-RF-negative arthritis, $40 \%$ would be expected to be ANA positive, and $10 \%$ to develop uveitis. $^{2} 45$

Results of APRs (CRP and ESR) were raised significantly less frequently in A-DS when compared to children with JIA, although ESR does appear to be more useful in aiding diagnosis of both conditions compared with CRP (figure 4B). This highlights the importance of a thorough history and examination when caring for children with suspected A-DS. Clinical signs can be extremely subtle and may include slight asymmetry or loss of end range of movement of the affected joint. This subtlety of clinical findings is often confounded by failure of children with DS to report pain on history or examination. Blood tests can be falsely reassuring and so a low threshold to refer for MRI with gadolinium contrast is advised in any suspected cases of A-DS. Our experience is that there was greater than expected MRI confirmation of active synovitis if correlating to clinical signs. In two of the four cases imaged with MRI although the history was suggestive of a possible previous inflammatory episode, there were no clinical signs to support the same on examination. MRI (with gadolinium contrast) is considered the gold standard for diagnosis of active synovitis. ${ }^{43}$ It is very helpful in detecting synovial abnormalities within the joint, and is more sensitive than clinical and radiographic examination for detection of inflammatory soft tissue and early bone changes. ${ }^{43}$ However, high cost, limited availability and the frequent need for sedation can limit the use of MRI.

We describe a cohort of children with arthritis and DS that most frequently present with a polyarticular-RF-negative arthritis, predominantly affecting the small joints of the hands and wrists. The arthritis is erosive in nature; a finding we do not believe is solely related to the observed delay in diagnosis of A-DS. We also report a lack of ANA positivity and often-minimal acute phase markers on review of blood results. It may be reasonable to suggest that A-DS is a distinct disease to JIA. Children with DS have different genetics and immunology than children 
without DS, and are at increased risk of a number of autoimmune conditions, further supporting this theory. ${ }^{12-22}$ Future studies to explore the immune, histological and genetic basis of A-DS are required to improve our understanding of the pathogenesis of A-DS.

Modern treatment of JIA involves rapid induction of disease control. At present, treatment for children with A-DS follows guidelines developed and implemented to treat JIA. However, this may not be best practice given the fact that we have identified a number of differences between the features of both arthritis cohorts. A detailed review of treatment instigated in our A-DS cohort and long-term disease outcome would be important to identify if this cohort respond to treatment as expected for children with JIA. This, combined with exploration of the immuno-histopathology of A-DS would enable identification of more targeted treatments for this cohort of children, providing a more personalised model of care, and in turn better clinical outcomes.

This study highlights the importance of raising awareness about the increased risk of arthritis in children with DS to aid better recognition and more timely diagnosis of A-DS. The addition of an annual musculoskeletal examination to the well-recognised health surveillance guidelines developed by the Down syndrome Medical interest group for all children with DS would be an initiative of great benefit to this cohort of children. As already stated, more work is required to better understand disease pathogenesis of A-DS and in turn identify best practice in terms of treatment guidelines for A-DS.

Finally, we propose a more appropriate clinical term that better reflects the inflammatory, erosive nature of the disease would be DS-associated arthritis (DA). In order to facilitate more cohesive clinical practice and future collaborative research, we would hope that this could become a universally accepted term among healthcare professionals and the general public.

A limitation to our study design was that we did not offer a similar musculoskeletal screening programme to children without DS. Therefore, our route to rheumatology results (figure 3) may not be totally representative of a typical JIA cohort. It is possible that if a similar screening programme were offered to children without DS, some undiagnosed cases of JIA would be detected. However, we predict that this number would not be as high as detected in children with DS given the fact that children without DS do not face the same challenges as outlined in figure 5 that contribute to failure to recognise and diagnose A-DS.

Acknowledgements Special thanks to Down Syndrome Ireland, the Chartered Physiotherapists in Intellectual Disability (CPID) and the children and families that participated in the study.

Contributors $\mathrm{Dr}$ CF and OGK are substantial contributions to the conception or design of the work; or the acquisition, analysis or interpretation of data for the work; and drafting the work or revising it critically for important intellectual content; and final approval of the version to be published; and agreement to be accountable for all aspects of the work in ensuring that questions related to the accuracy or integrity of any part of the work are appropriately investigated and resolved. EJMacD and DD are revising the work critically for important intellectual content; and final approval of the version to be published.

Funding This work was supported by the National Children's Research Centre, Crumlin, Dublin; grant number D/13/4.

Competing interests None declared.

Patient consent for publication Not required.

Provenance and peer review Not commissioned; externally peer reviewed.

Data sharing statement All data relevant to the study are included in the article or uploaded as supplementary information.

Open access This is an open access article distributed in accordance with the Creative Commons Attribution Non Commercial (CC BY-NC 4.0) license, which permits others to distribute, remix, adapt, build upon this work non-commercially, and license their derivative works on different terms, provided the original work is properly cited, appropriate credit is given, any changes made indicated, and the use is non-commercial. See: http://creativecommons.org/licenses/by-nc/4.0/.

\section{REFERENCES}

1. Petty E, Southwood TR, Manners P, et al. International League of associations for rheumatology classification of juvenile idiopathic arthritis: second revision, Edmonton, 2001. Journal of Rheumatology 2004;31:390-2.

2. Hayward K, Wallace CA. Recent developments in anti-rheumatic drugs in pediatrics: treatment of juvenile idiopathic arthritis. Arthritis Res Ther 2009;11.

3. Thierry S, Fautrel B, Lemelle I, et al. Prevalence and incidence of juvenile idiopathic arthritis: a systematic review. Joint Bone Spine 2014:81:112-7.

4. Nell VPKet al. Benefit of very early referral and very early therapy with disease-modifying anti-rheumatic drugs in patients with early rheumatoid arthritis. Rheumatology 2004;43:906-14.

5. Foster HE, Eltringham MS, Kay LJ, et al. Delay in access to appropriate care for children presenting with musculoskeletal symptoms and ultimately diagnosed with juvenile idiopathic arthritis. Arthritis Rheum 2007;57:921-7.

6. Albers HM, Wessels JAM, van der Straaten RJHM, et al. Time to treatment as an important factor for the response to methotrexate in juvenile idiopathic arthritis. Arthritis Rheum 2009;61:46-51.

7. Magnani A, Pistorio A, Magni-Manzoni S, et al. Achievement of a state of inactive disease at least once in the first 5 years predicts better outcome of patients with polyarticular juvenile idiopathic arthritis. The Journal of Rheumatology 2009;36:628-34.

8. Gowdie PJ, Tse SML, Arthritis Jl. Juvenile idiopathic arthritis. Pediatric Clinics of North America 2012;59:301-27.

9. Packham JC, Hall MA. Long-term follow-up of 246 adults with juvenile idiopathic arthritis: functional outcome. Rheumatology 2002:41:1428-35.

10. Epstein CJ. Down Syndrome (trisomy 21). In: Scriver CR, Beaudet AL, Sly WS, et al, eds. The metabolic and molecular bases of inherited diseases. New York: McGraw-Hill, Inc, 2001: 1223-56.

11. Nelson MR. Pediatrics. New York: Demos Medical Publishing, 2011.

12. Hickey F, Hickey E, Summar KL. Medical update for children with Down syndrome for the pediatrician and family practitioner. Adv Pediatr 2012;59:137-57.

13. Mandal K. Down syndrome. In: Ghosh A, Mitra M, Choudhury J, eds. Treatment and prognosis in pediatrics. Jaypee Brothers Medical Publishers Ltd, 2013: 391.

14. Peiper S, Strayer DS. Developmental and Genetic Diseases. In Rubin E, Reisner HM, eds. Essentials of Rubin's Pathology. 6th edn. Lippincott: Williams and Wilkins, 2013.

15. Asim A, Kumar A, Muthuswamy $S$, et al. "Down syndrome: an insight of the disease". Journal of Biomedical Science 2015;22.

16. Johnson Z, Lillis D, Delany V, et al. The epidemiology of Down syndrome in four counties in Ireland 1981-1990. Journal of Public Health 1996;18:78-86

17. Weijerman ME, de Winter JP. Clinical practice. the care of children with Down syndrome. Eur J Pediatr 2010;169:1445-52.

18. Jeremiah DE, Leyshon GE, Francis TRHWS, et al. Down's syndrome and diabetes. Psychological Medicine 1973;3:455-7.

19. Loudon MM, Day RE, Duke EM. Thyroid dysfunction in Down's syndrome. Archives of Disease in Childhood (1985;60:1149-51.

20. Jansson $U$, Johansson $C$. Down syndrome and celiac disease. $J$ Pediatr Gastroenterol Nutr 1995;21:443-5. and.

21. Gale L, Wimalaratna H, Brotodiharjo A, et al. Down's syndrome is strongly associated with coeliac disease. Gut 1997;40:492-6. 
22. Van Goor JC, Massa GG, Hirasing R. Increased incidence and prevalence of diabetes mellitus in Down's syndrome. Arch Dis Child $1997 ; 77$.

23. Torfs CP, Christianson RE. Anomalies in Down syndrome individuals in a large population-based registry. American Journal of Medical Genetics 1998;77:431-8.

24. Fudge JC, Li S, Jaggers J, et al. Congenital heart surgery outcomes in Down syndrome: analysis of a national clinical database. PEDIATRICS 2010;126:315-22.

25. Shrestha M, Shrestha $U$, Shakya U. Down syndrome and congenital heart disease: single centre, prospective study. Nepal Journal of Medical Sciences 2013;2:96-101.

26. Juj $\mathrm{H}$, Emery $\mathrm{H}$. The arthropathy of Down syndrome: an underdiagnosed and under-recognized condition. J Pediatr 2009;154:234-8.

27. Sherk HH, Pasquariello PS, Watters WC. Multiple Dislocations of the Cervical Spine in a Patient with Juvenile Rheumatoid Arthritis and Down??s Syndrome. Clin Orthop Relat Res 1982;162:37-40.

28. Herring JA, Fielding WJ. Cervical instability in Down's syndrome and juvenile rheumatoid arthritis. J Pediatr Orthop 1982;2:205-7.

29. Padmakumar B, Evans Jones LG, Sills JA. Is arthritis more common in children with Down syndrome? Rheumatology 2002;41:1191-3.

30. Yancey CL, Zmijewski C, Athreya BH, et al. Arthropathy of Down's syndrome. Arthritis Rheum 1984;27:929-34.

31. Olson JC, Bender JC, Levinson JE, et al. Arthropathy of Down syndrome'. Pediatrics 1990;1990:931-6.

32. Jones JT, Talib N, Lovell D, et al. Clinical features and treatment of Down syndrome arthropathy: experience from two us tertiary hospitals. Pediatr Drugs 2019;21:33-9.

33. Aoust L, Rossi-Semerano L, Koné-Paut I, et al. Time to diagnosis in juvenile idiopathic arthritis: a French perspective. Orphanet J Rare Dis 2017;12.
34. Foster H, Rapley T, May C. Juvenile idiopathic arthritis: improved outcome requires improved access to care. Rheumatology 2010;49:401-3. and.

35. Elliott D, Gray S, Weeks DJ. Verbal Cuing and motor skill acquisition for adults with Down syndrome. Adapted Physical Activity Quarterly 1991;8:210-20.

36. Palisano RJ, Walter SD, Russell DJ, et al. Gross motor function of children with Down syndrome: creation of motor growth curves. Arch Phys Med Rehabil 2001;82:494-500.

37. Malak R, Kostiukow A, Krawczyk-Wasielewska A, et al. Delays in motor development in children with Down syndrome. Med Sci Monit 2015;21:1904-10.

38. Cruikshank M, Tunc A, Walsh J, et al. Arthritis in Down's syndrome is still being missed. Pediatric Rheumatology 2008;6.

39. Martínez-Cué $\mathrm{C}$, Baamonde $\mathrm{C}$, Lumbreras MA, et al. A murine model for Down syndrome shows reduced responsiveness to pain. Neuroreport 1999;10:1119-22.

40. Hennequin M, Morin C, Feine JS. Pain expression and stimulus localisation in individuals with Down's syndrome. The Lancet 2000;356:1882-7.

41. Hennequin M, Faulks D, Allison PJ. Parents' ability to perceive pain experienced by their child with Down syndrome. J Orofac Pain 2003;17:347-53.

42. Defrin R, Pick CG, Peretz C, et al. A quantitative somatosensory testing of pain threshold in individuals with mental retardation. Pain 2004;108:58-66.

43. Petty R, Laxer R, Lindsley C, et al. Textbook of paediatric rheumatology. 7th edn. Saunders, 2015.

44. Greenwald AG, Zakerzadeh A, Laxer RM, et al. Later-onset rheumatoid factor negative polyarticular juvenile idiopathic arthritis (JIA): a unique patient group? Clin Exp Rheumatol 2013;31:645-52.

45. Saurenmann RK, Levin AV, Feldman BM, et al. Prevalence, risk factors, and outcome of uveitis in juvenile idiopathic arthritis: a longterm followup study. Arthritis Rheum 2007;56:647-57. 